\title{
USE OF CLOSED SYSTEM OF AIR THROUGH EARTH TUBES AS AN ENVIRONMENT FRIEND FOR HEATING GREENHOUSES
}

Alghannam, A. 0 .

Agric. Systems Eng. Dept., College of Agric. and Food Sci., King Faisal Univ. Saudi Arabia.

Email: aalghannam@kfu.edu.sa

استخدام الدفع الموجب للهواء الطبيعي في أنابيب أرضية لتدفئة البيوت المحمية

قسم هندة النظم الزراعية، كلية العلوم الزراعية والأغذية، جامعة الملك فيصل، المملكة العربية السعودية الرئية aalghannam@kfu.edu.sa البريد الإكترونية

$$
\text { ألملخص }
$$

يهذف هذا البحث إلى دراسة استخدام النظام المغلق للهواء خلال الأنابيب الأرضية في تدفئة البيوت

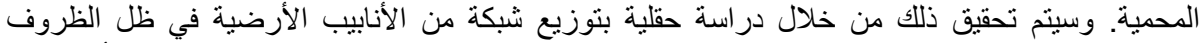

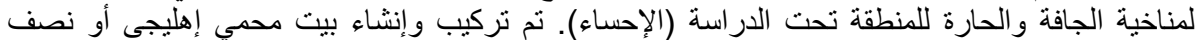

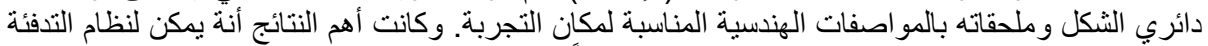

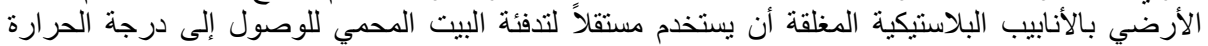

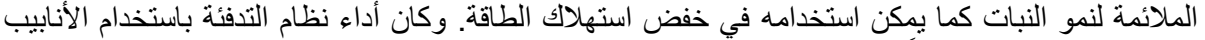

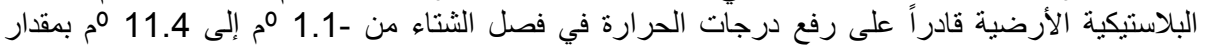

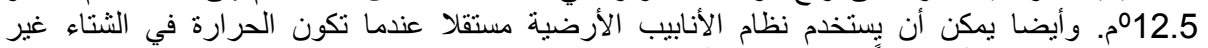

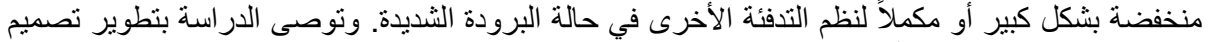

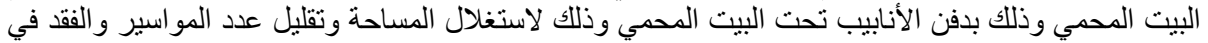
درجات الحرارة.

\section{المقدمة}

تعتبر عملية التدفئة والمحافظة على درجة الحرارة داخل البيوت المحمية من أهم العو امل الرئيسية في

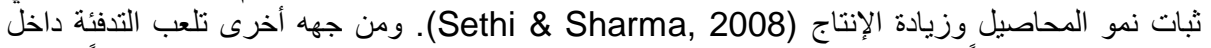

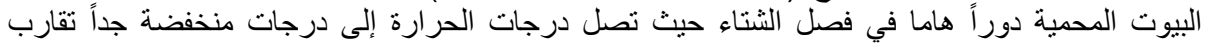

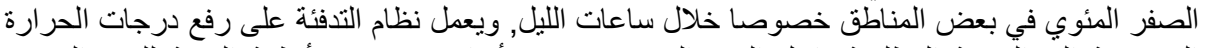

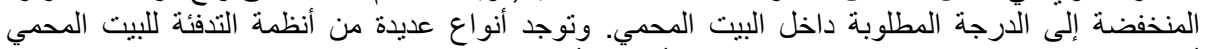

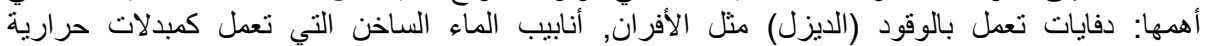

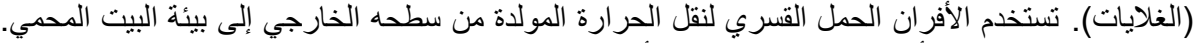

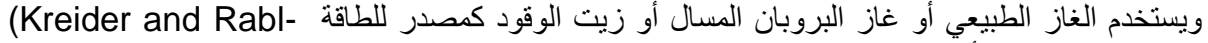

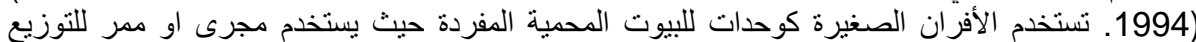

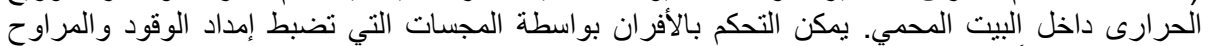

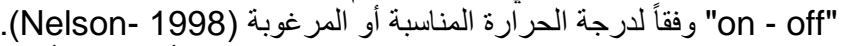

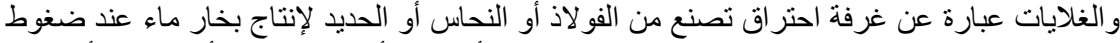

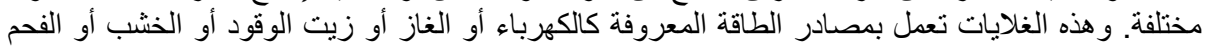

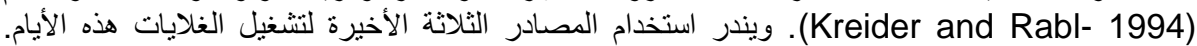

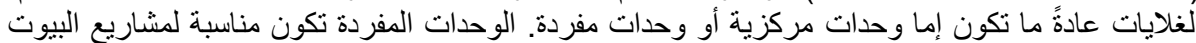

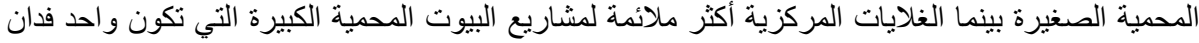


Alghannam, A. 0 .

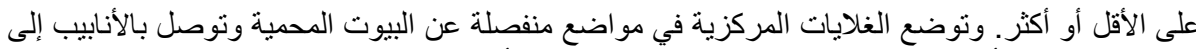

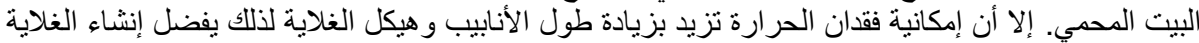

داخل البيت المحمي (Nelson-1998).

تحول المدفأة المشعة الأشعة تحت الحمر اء المتصة بو بواسطة أي جسم في طريقها وتسخنه ومن ثم هذه

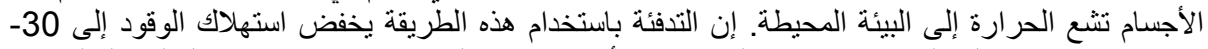

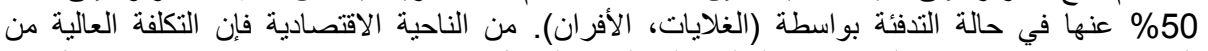

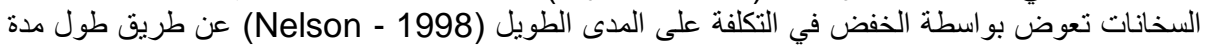

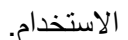

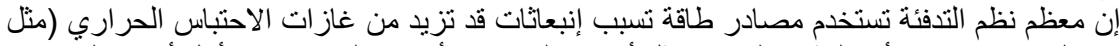
ثاني أكسيد الكربون (CO

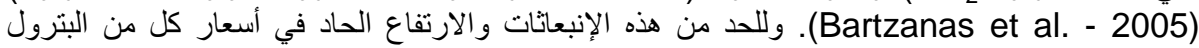

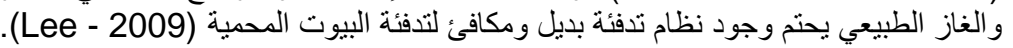

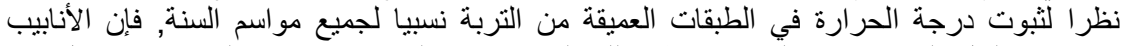

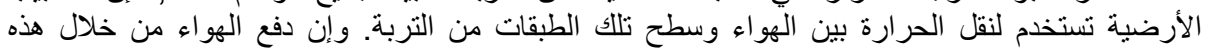
الأنابيب سيوز عن من خلال الفتحات و المخارج إلى البيت الكيت المحمي.

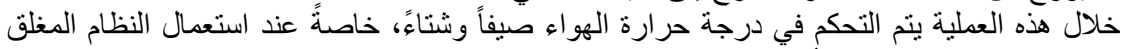

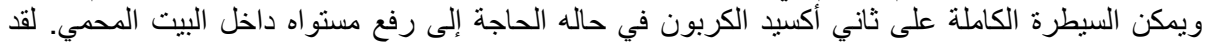

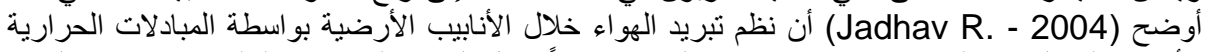

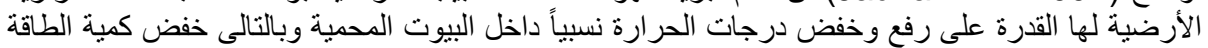

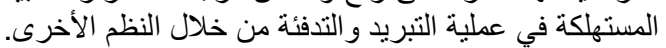
تمت دراسة تطوير تصاميم مختلفة لأنظمة الأنابيب الأرضية في عملية التبريد و التدفئة للبيوت

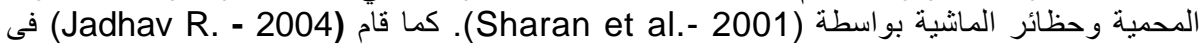

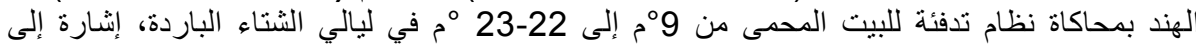
تقليل 34 \% من ماء الري، وزيادة كمية الدحصول نحو 2.7 مرة مقارنة بالحقل فئل المفتوح نتيجة لثبات

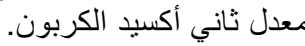

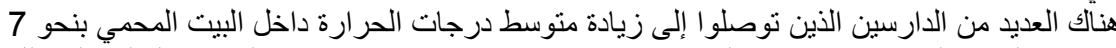

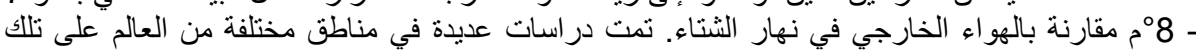

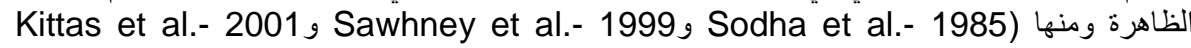
(Lee and Short- 2001,

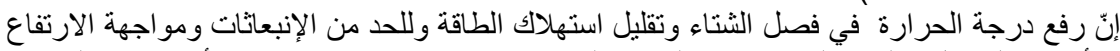

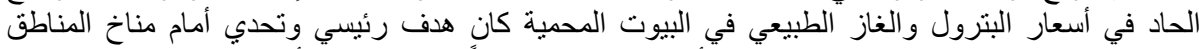

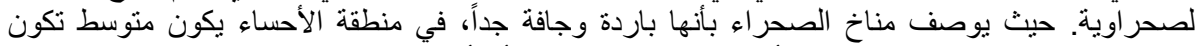

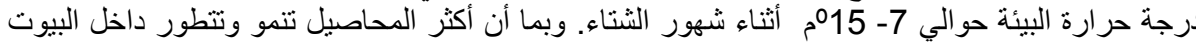

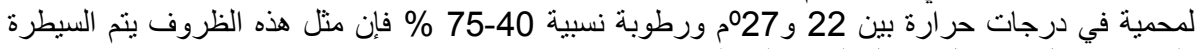

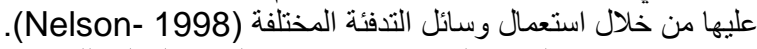

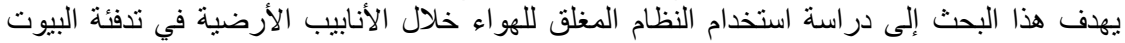

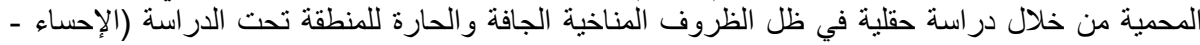
المنطقة الغربية - السعودية).

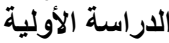

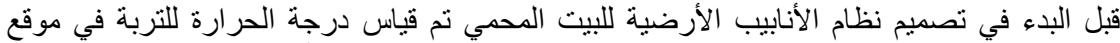

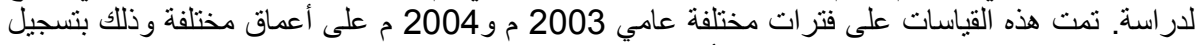

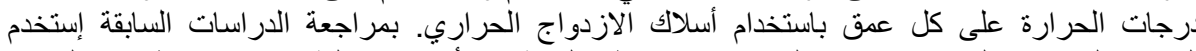

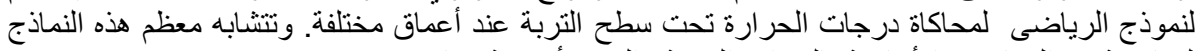

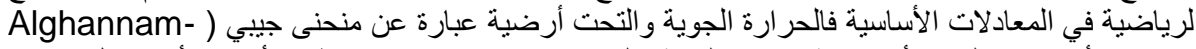

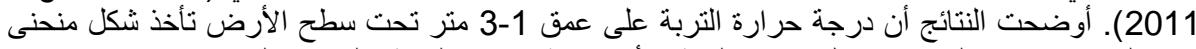

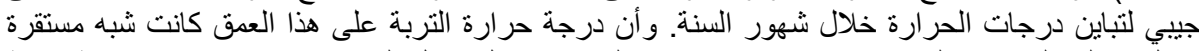

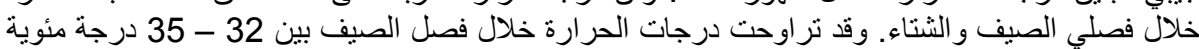
وبين 19-20 درجة مئوية خلال فصل الثناء فى منطقة الدراسة. 


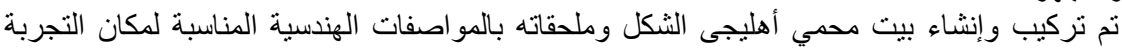

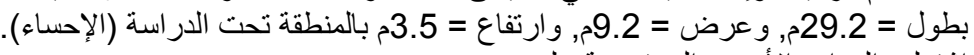

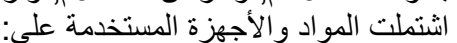

مروحة طرد مركزية (القدرة 1.59 حصان و عدد الإدة اللفات في الدقيقة 1402 لفة ــ تدفق الهو اء 5000

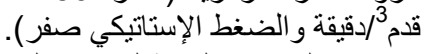
وصلات المواسير المرنة لممرات الهيكئن الهواء.

- - مجسات HOBO data logger لرصد وتسجيل درجات الحرارة و الرطوبة داخل وخارج البيت المحمي و البيت المقارن.

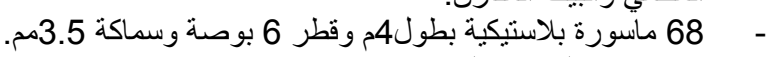

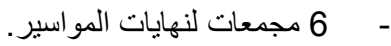

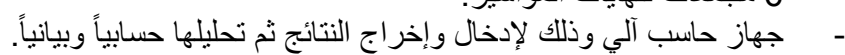

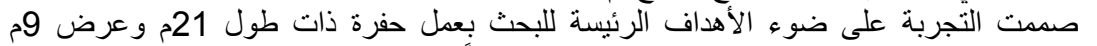

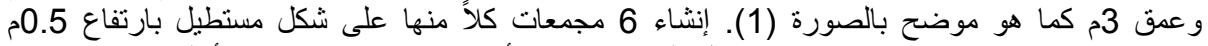

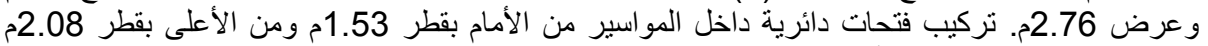

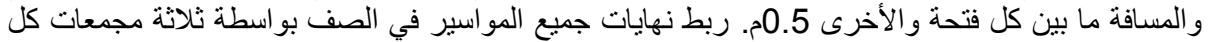

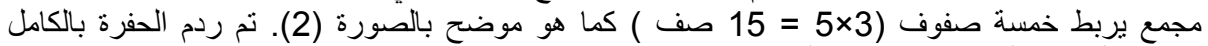

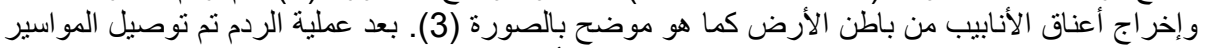

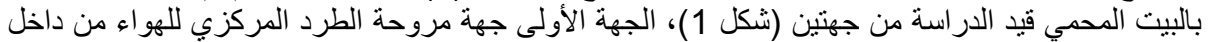

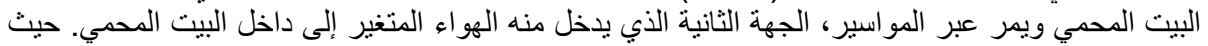

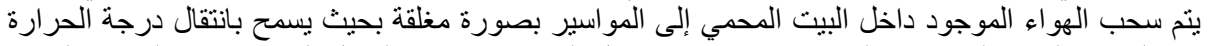

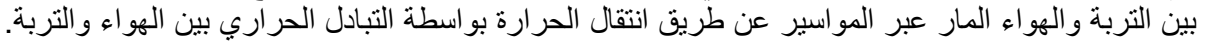

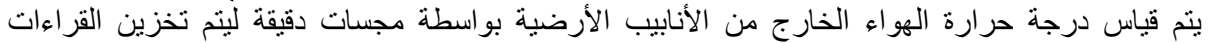

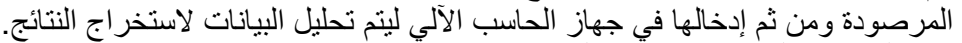

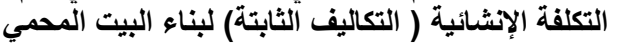

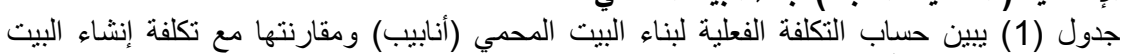

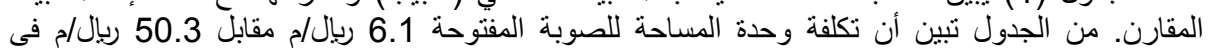

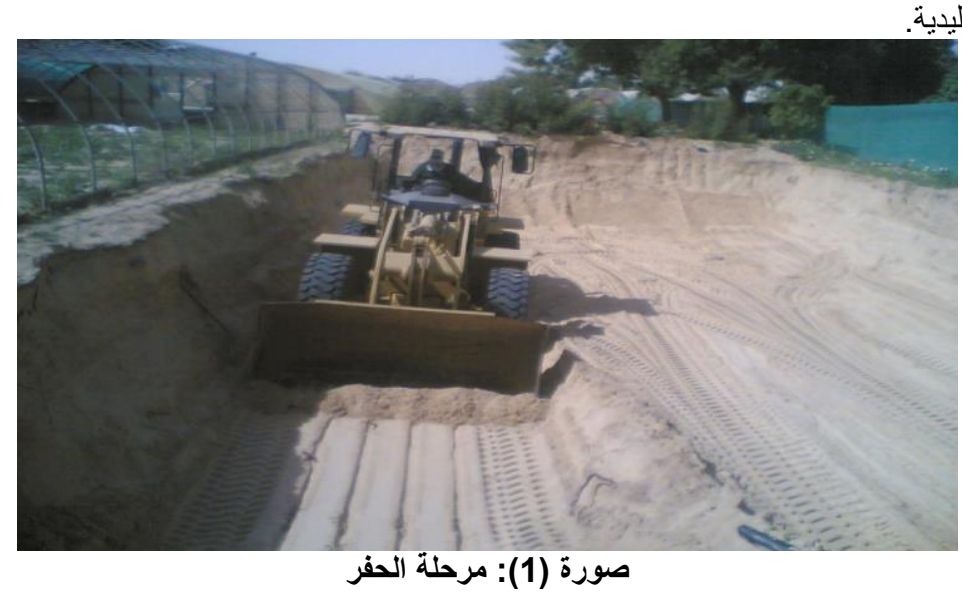


Alghannam, A. 0.

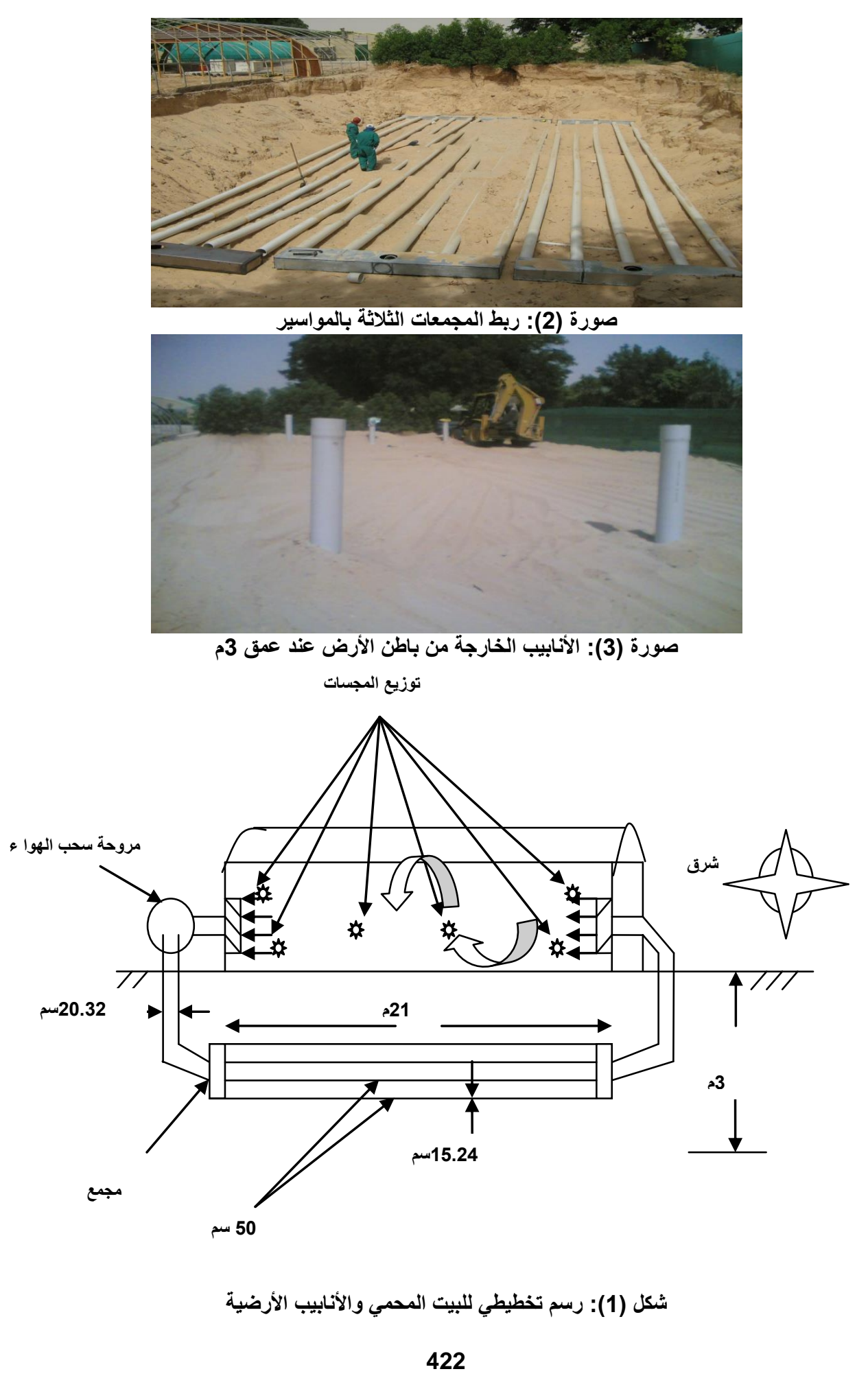




\section{النتائج والمناقشة}

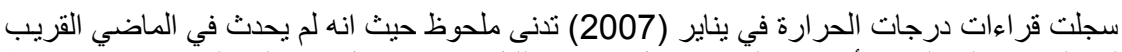

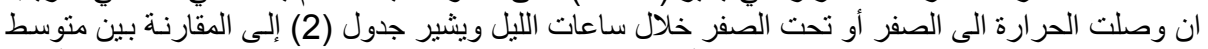

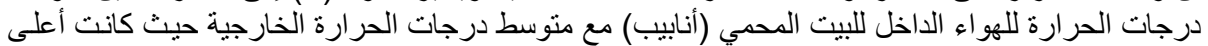

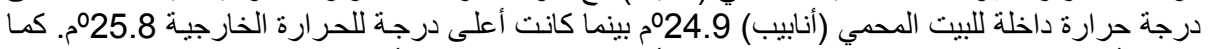

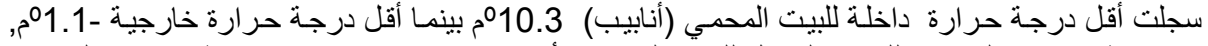

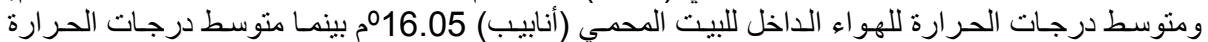

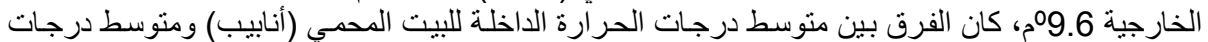

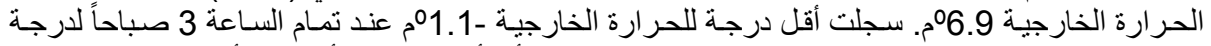

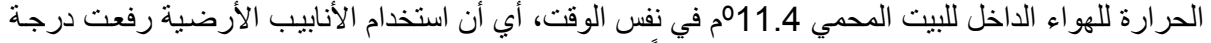

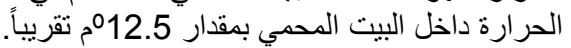

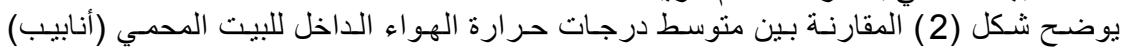

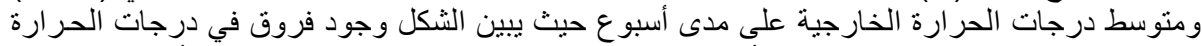

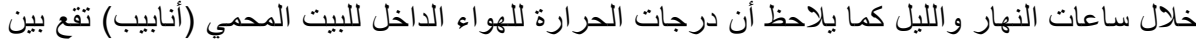

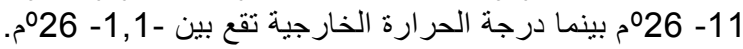

جدول (1): تكلفة إنشاء البيث المحمي تبريد أنابيب والبيث المحمي تبريد تبخيري

\begin{tabular}{|c|c|c|c|}
\hline \multicolumn{2}{|c|}{ البيت المحمي تبريد تبخيري } & \multicolumn{2}{|c|}{ البيت المحمي تبريد أنابيب } \\
\hline | التكلفة بالريال & اسم الصنف & التكلفة بالريال & اسم الصنف \\
\hline 5500 & وسائد & 6700 & أنابيب بلاستيكية \\
\hline 3600 & |مروحة & 5000 & مروحة طرد مركزية \\
\hline 2750 & غطاء بلاستيكي & 2750 & غطاء بلاستيكي \\
\hline 300 & |مضخة مباه & 800 & حفز \\
\hline 250 & خز ان مباه بعو امة & 1000 & عمال \\
\hline 300 & |مو اسير لإيصال الماء للخلايا & - & - \\
\hline 800 & 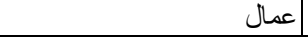 & & \\
\hline 13500ربيال = 50.3 ريال/ה & |مجموع التكلفة & 625 ربال = 6.1 ربال/م & مجموع التكلفة \\
\hline
\end{tabular}

جدول (2):الفرق بين متوسط درجات الجرارة الداخلية ومتوسط درجات الحرارة الخارجية في فصل الثتاء

\begin{tabular}{|c|c|c|c|c|c|}
\hline $\begin{array}{c}\text { الاختلاف } \\
\text { (CV, \%) }\end{array}$ & 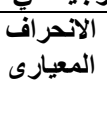 & متوسط درجة & أعلى درجة & حرارة (أقمّم) & وجه المقارنة \\
\hline 22.1 & $3.6 \pm$ & 16.05 & 24.9 & 10.3 & (أنابيب) الحرارة للهواء الداخل للبيت المحمي \\
\hline 64.4 & $6.8 \pm$ & 10.6 & 28.6 & 0.4 & داخل البيت المحمي (مقارن) \\
\hline 72.3 & $6.9 \pm$ & 9.6 & 25.8 & $1.1-$ & درجة الحرارة الخارجية \\
\hline
\end{tabular}

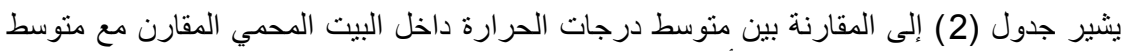

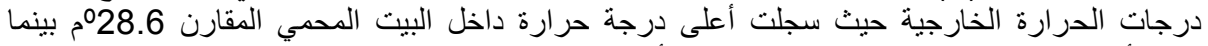

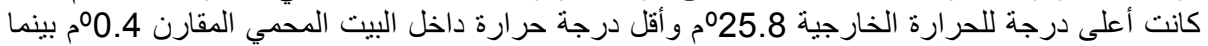

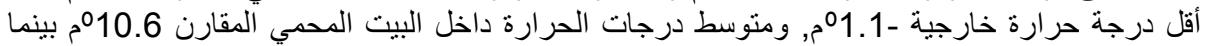

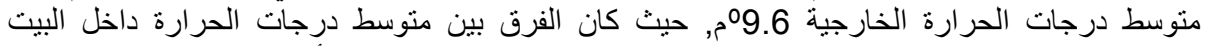

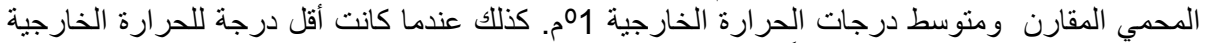
-1.10 الوقت حيث تم رفع درجة الحرارة بمقدار 

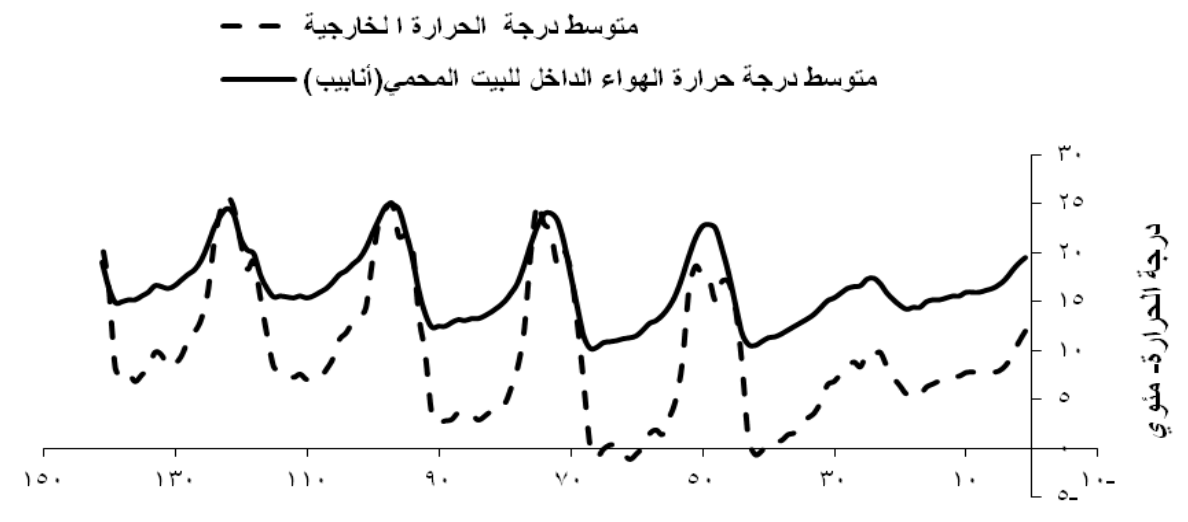

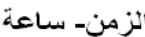

شكل (2) المقارنـة بين متوسط درجة حرارة الهواء الداخل للبيت المحمي (أنابيب) مـع درجة الحرارة

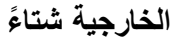

شكل (3) ييين المقارنة بين متوسط درجات الحرارة الداخلية (دقارن) ومتوسط درجات الحرارة الخارجية

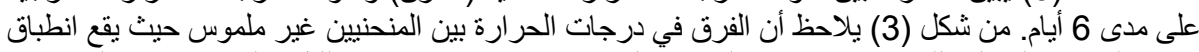

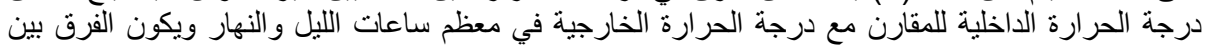

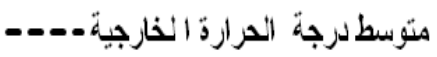
المنحنيين متقارب في التلث الأخير من الليل.

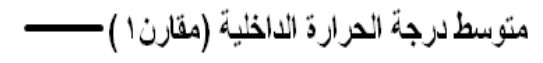

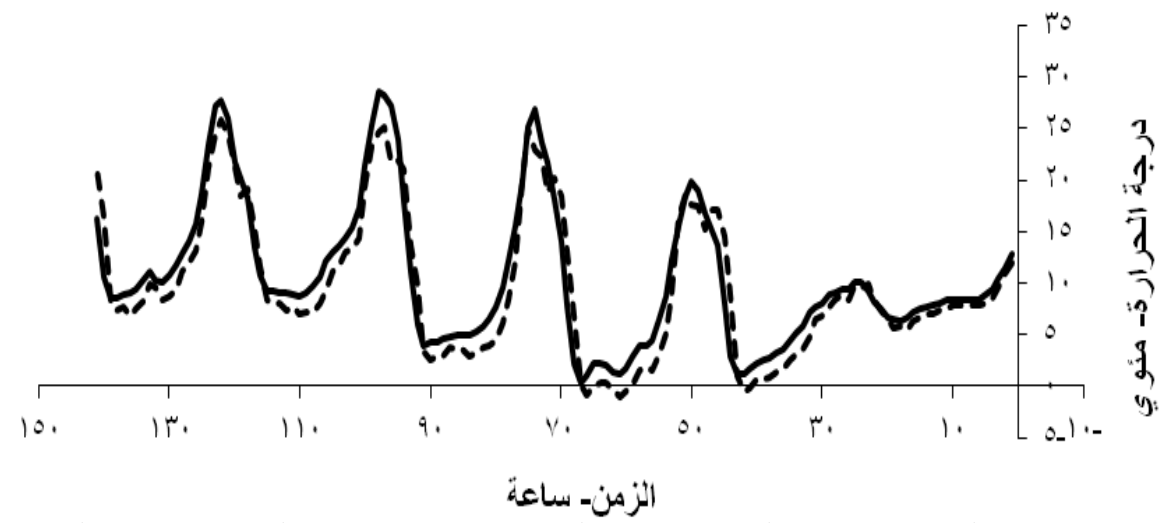

شكل (3): المقارنة بين متوسط درجة الحرارة الداخلية (مقارن) مع متوسط درجة الحرارة الخارجية.

يثير جدول (3) إلى المقارنة بين منوسط درجات الحرارة الداخلة ومتوسط درجات الحرارة الخارجة

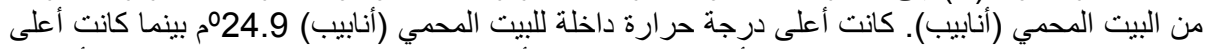

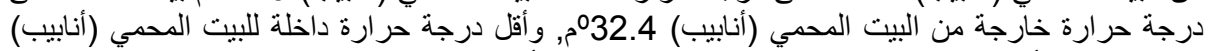

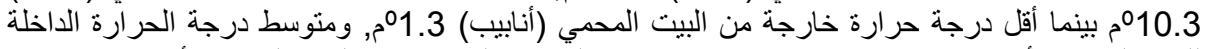

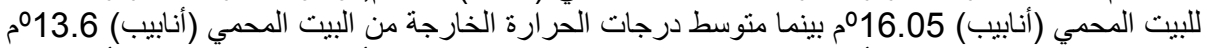

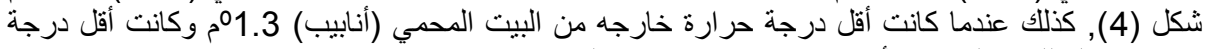

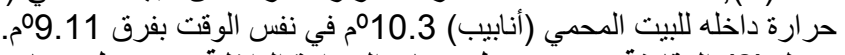

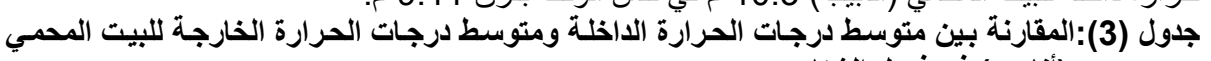
(أنابيب) في فصل الثتاء . 
J. Soil Sci. and Agric. Eng., Mansoura Univ., Vol. 3 (3), March, 2012

\begin{tabular}{|c|c|c|c|c|c|}
\hline $\begin{array}{c}\text { الاختلاف } \\
\text { (CV, \%) }\end{array}$ & الالحيارى اف & متوسط درجة (الحرة) & أعلى درجة & 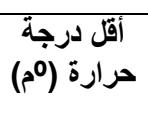 & وجه المقارنة \\
\hline 57.7 & $7.8 \pm$ & 6 & 32.4 & 1.3 & ة الحرارة الخارجة (أنابيب) \\
\hline 22.1 & $3.6 \pm$ & 16.05 & 24.9 & 10.3 & جة الحرارة الداخلة (أنابيب) \\
\hline
\end{tabular}

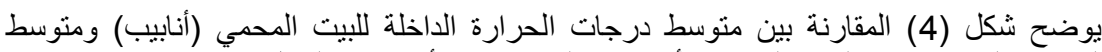

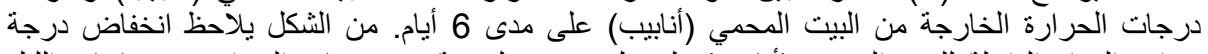

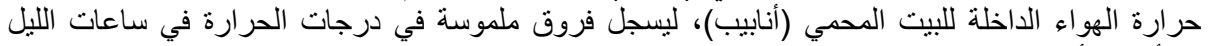

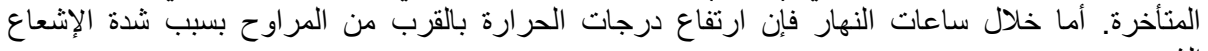

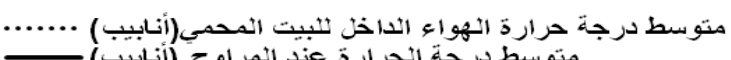

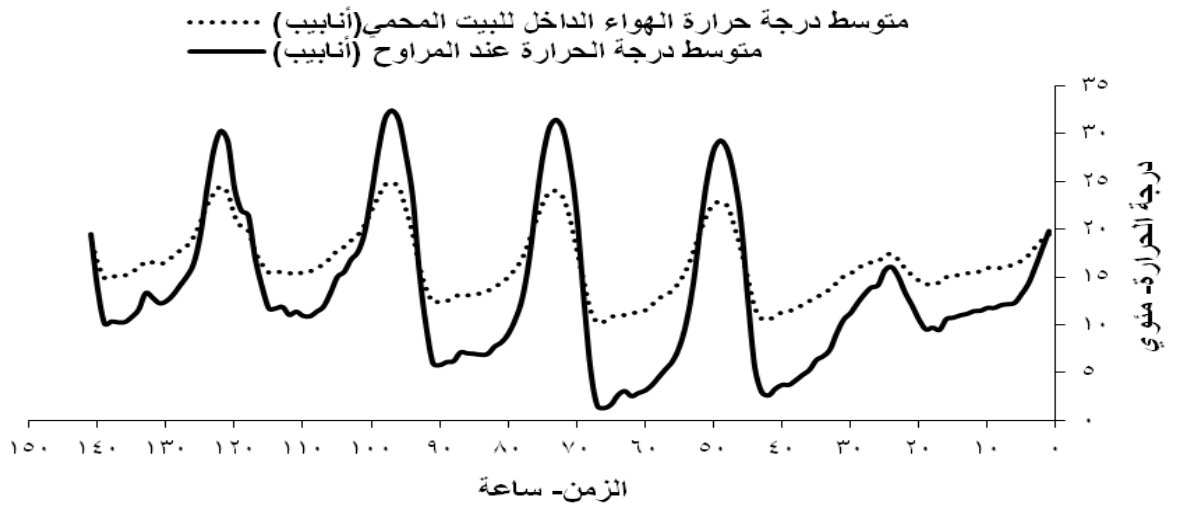

شكل (4): منحنى يوضح المقارنة بين متوسط درجة الحرارة الاخلة والخارجة (أنابيب)

يوضح الجدول (4) المقارنة في الرطوبة النسبية بين البيت المحمي (أنابيب) والبيت المحمي الدقارن

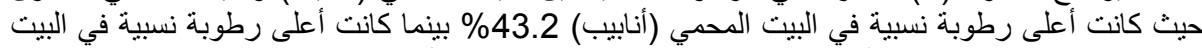

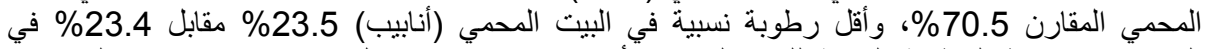

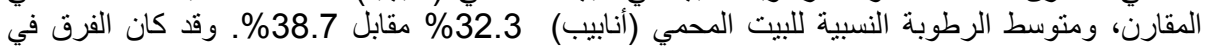

متوسط الرطوبة النسبية بين البيت المحمي ( أنابيب) والبيت الدحمي المقارن حوالى 6.4\% \%

جدول رقم (4) المقارنة بين الرطوبة النسبية في البيت المحمي (أنابيب) و(المقارن) شتاءُ.

\begin{tabular}{|c|c|c|c|c|c|}
\hline $\begin{array}{c}\text { الاختلاف } \\
\text { (CV, \% }\end{array}$ & الانحيارى & نسبية \% & نسبية رطوبة & أفبية رطوبة & وجه المقارنة \\
\hline 14.7 & $4.8 \pm$ & 32.3 & 43.2 & 23.5 & الرطوبة النسبية (أنابيب) \\
\hline 32.5 & $12.6 \pm$ & 38.7 & 70.5 & 23.4 & الرطوبة النسبية للمقارن \\
\hline
\end{tabular}

يوضح شكل (5) المقارنة بين معدل الرطوبة النسبية للبيت المحمي (أنابيب) و البيت المحمي المقارن

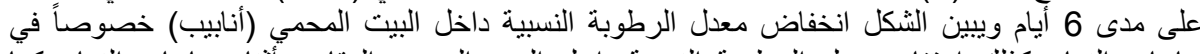

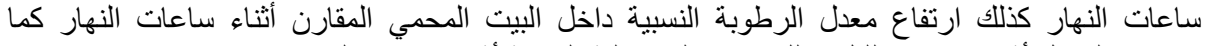
ينخفض المعدل أثناء ساعات الليل وذلك راجع إلى عملية التهوية أثناء ساعات النهات النهار. 


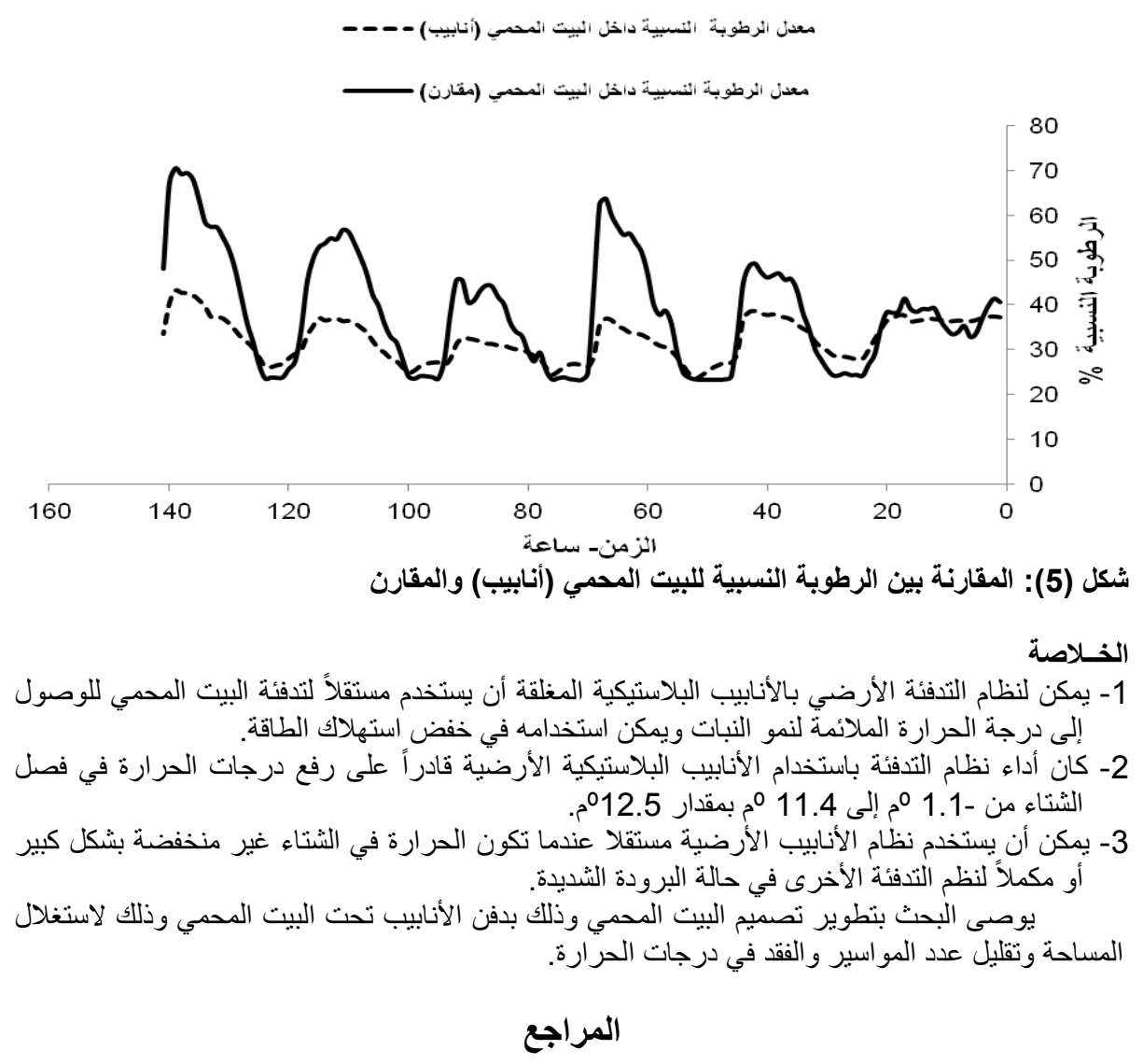

Alghannam, A.O. (2011). Mathematical Simulation of soil Subsurface Temperatures, Minufiya J. Agric. Res. Vol.36 No. 3

Bartzanas T., Tchamitchian M. and C. Kittas (2005). Influence of the Heating Method on Greenhouse Microclimate and Energy Consumption. Biosystems Engineering (2005) 91 (4), 487-499.

Jadhav R. (2004). Performance of Greenhouse Coupled to Earth-TubeHeat-Exchanger in Closed-Loop Mode.

Kittas C., Bartzanas T. and A. Jaffrin (2001). Greenhouse Evaporative Cooling: Measurement and Data Analysis, Transactions of the ASAE Vol. 44(3): 683-689.

Kreider J. and A. Rabl. (1994). Heating and Cooling Buildings. McGraw-Hill, Inc. NJ.

Lee I. B. and T. H. Short (2001). Verification of Computational Fluid Dynamic Temperature Simulations in Full-Scale Naturally Ventilated Greenhouse, Transactions of the ASAE .V.44 (1): 119-127.

Lee J. (2009). Current status of ground source heat pumps in Korea. Renewable and Sustainable Energy Reviews, 13, 1560e1568

Nelson, P. V. (1998). Greenhouse Operation and Management. Prentice-Hall, Inc. Upper Saddle River, NJ 07458. 
Sawhney R. L. and D. Buddhi (1999). Thanu NM. An experimental study of summer performance of a recirculation type underground airpipe air conditioning system. Building and Environment 1999;34:189-96

Sethi V.P. and S. K. Sharma (2008). Survey and evaluation of heating technologies for worldwide agricultural greenhouse applications. Solar Energy 82: 832-859.

Sharan G.; Sahu R.K. and Jadhav R. (2001). Earth-tube heat exchanger based airconditioning

Sodha M. S.; Sharma A. K.; and S. P. Singh (1985). Bansal Kumar A. Evaluation of an earth-air tunnel system for cooling/heating of a hospital complex. Building and Environment 1985; 20(2): 115-22.

\title{
USE OF CLOSED SYSTEM OF AIR THROUGH EARTH TUBES AS AN ENVIRONMENT FRIEND FOR HEATING GREENHOUSES \\ Alghannam, A. 0 . \\ Agric. Systems Eng. Dept., College of Agric. and Food Sci., King Faisal Univ. Saudi Arabia. \\ Email: aalghannam@kfu.edu.sa
}

\begin{abstract}
This research aims to study the use of the closed system of air through earth tubes for heating greenhouses. This had been achieved by a field study using plastic earth tubes under the hot climatic conditions of the region under study. An elliptical shaped greenhouse and its accessories were constructed according to the appropriate engineering specifications of the experiment site. Results showed that the heating closed systems of air through plastic earth tubes can be used independently for heating the greenhouse in order to attain the suitable temperature for plant growth. The performance of the heating system was capable to increase temperatures during winter season from $-1.1^{\circ} \mathrm{C}-11.4^{\circ} \mathrm{C}$ i.e. by a magnitude of $12.5^{\circ} \mathrm{C}$. Also the system can be used independently when the temperature during winter is not substantially reduced or as a complementary to other heating systems in case of extreme cold.

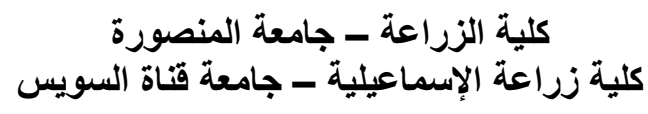

كلية الزراعة - جامعة المنصورة

كلية زراعة الإسماعيلية - جامعة فئاة السويس

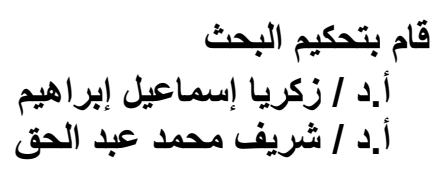

قام بتحكيم البحث

أ. أد / أدكريا إسماعيل إبراهيم

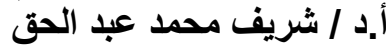

